\title{
Hybrid Energy Efficient Distributed Protocol for Heterogeneous Wireless Sensor Network
}

\author{
Harneet Kour \\ Department of Computer Science and Engineering \\ National Institute of Technology Jalandhar, \\ Punjab, India.
}

\author{
Ajay K. Sharma \\ Department of Computer Science and Engineering \\ National Institute of Technology Jalandhar, \\ Punjab, India.
}

\begin{abstract}
The main requirements of wireless sensor network are to prolong the network lifetime and energy efficiency. In this paper, Heterogeneous - Hybrid Energy Efficient Distributed Protocol (H-HEED) for Wireless Sensor Network has been proposed to prolong the network lifetime. In this paper the impact of heterogeneity in terms of node energy in wireless sensor networks have been mentioned. Finally the simulation result demonstrates that H-HEED achieves longer lifetime and more effective data packets in comparison with the HEED protocol.
\end{abstract}

\section{Keywords}

Wireless Sensor Network, Network Lifetime, Heterogeneity.

\section{INTRODUCTION}

A wireless sensor network (WSN) can be defined as a network consists of low-size and low-complex devices called as sensor nodes that can sense the environment and gather the information from the monitoring field and communicate through wireless links; the data collected is forwarded, via multiple hops relaying to a sink (also called as controller or monitor) that can use it locally, or is connected to other networks [1]. A sensor node usually consists of four sub-systems [2] i.e. sensing unit, processing unit, communication unit and power supply unit.

In WSN, the sensor nodes are deployed in a sensor field. The deployment of the sensor nodes can be random (i.e. dropped from the aircraft), regular (i.e. well planned or fixed) or mobile sensor nodes can be used. Sensor nodes coordinate among themselves to produce high-quality information about the physical environment. Each sensor node bases its decisions on its mission, the information it currently has, and its knowledge of its computing, communication, and energy resources. Each sensor nodes collect the data and route the data to the base station. All of the nodes are not necessarily communicating at any particular time and nodes can only communicate with a few nearby nodes. The network has a routing protocol to control the routing of data messages between nodes. The routing protocol also attempts to get messages to the base station in an energy-efficient manner.

The base station is a master node. Data sensed by the network is routed back to a base station. The base station is a larger computer where data from the sensor network will be compiled and processed. The base station may communicate with the Remote Controller node via Internet or Satellite [2, 3]. Human operators controlling the sensor network send commands and receive responses through the base station.
HEED (Hybrid Energy Efficient Distributed) protocol [4] is the clustering protocol. It uses using residual energy as primary parameter and network topology features (e.g. node degree, distances to neighbors) are only used as secondary parameters to break tie between candidate cluster heads, as a metric for cluster selection to achieve load balancing. In this all nodes are assumed to be homogenous i.e. all sensor nodes are equipped with same initial energy. But, in this paper we study the impact of heterogeneity in terms of node energy. We assume that a percentage of the node population is equipped with more energy than the rest of the nodes in the same network - this is the case of heterogeneous sensor networks. As the lifetime of sensor networks is limited there is a need to re-energize the sensor network by adding more nodes. These nodes will be equipped with more energy than the nodes that are already in use, which creates heterogeneity in terms of node energy, leads to the introduction of H-HEED protocol.

The remainder of the paper is organized as follows. In Section 2, we briefly review related work. Section 3 describes the clusters formation in the HEED protocol. Section 4 describes heterogeneous H-HEED protocol. Section 5 shows the performance of H-HEED by simulations and compares it with HEED. Finally, Section 6 gives concluding remarks.

\section{RELATED WORK}

W. R. Heinzelman, A. P. Chandrakasan and H. Balakrishnan [5] proposed Low Energy Adaptive Clustering Hierarchy (LEACH) protocol in 2000. It is one of the most popular hierarchical routing algorithms for sensor networks. The idea is to form clusters of the sensor nodes based on the received signal strength and use local cluster heads as routers to the sink. This will save energy since the transmissions will only be done by such cluster heads rather than all sensor nodes. Optimal number of cluster heads is estimated to be $5 \%$ of the total number of nodes. All the data processing such as data fusion and aggregation are local to the cluster. Cluster heads change randomly over time in order to balance the energy dissipation of nodes. This decision is made by the node choosing a random number between 0 and 1 . The node becomes a cluster head for the current round if the number is less than the following threshold:

$T(n)=\left\{\begin{array}{cc}\frac{p}{1-p n\left[\left(y m o d^{1}-p\right]\right.} & \text { if } n \in G \\ 0 & \text { otherwise }\end{array}\right.$

where $\mathrm{p}$ is the desired percentage of cluster heads (e.g. 0.05), $\mathrm{r}$ is $=$ the current round, and $\mathrm{G}$ is the set of nodes that have not been cluster heads in the last $1 / \mathrm{p}$ rounds. 
S. Lindsey and C. Raghavendra [6] introduced Power Efficient Gathering in Sensor Information Systems (PEGASIS) protocol in 2002. It is an improved version of LEACH. Instead of forming clusters, it is based on forming chains of sensor nodes. One node is responsible for routing the aggregated data to the sink. Each node aggregates the collected data with its own data, and then passes the aggregated data to the next ring. The difference from LEACH is to employ multi hop transmission and selecting only one node to transmit to the sink or base station. Since the overhead caused by dynamic cluster formation is eliminated, multi hop transmission and data aggregation is employed, PEGASIS outperforms the LEACH. However excessive delay is introduced for distant nodes, especially for large networks and single leader can be a bottleneck.

In 2001, A. Manjeshwar and D. P. Agarwal [7] proposed Threshold sensitive Energy Efficient sensor Network Protocol (TEEN) protocol. Closer nodes form clusters, with a cluster heads to transmit the collected data to one upper layer. Forming the clusters, cluster heads broadcast two threshold values. First one is hard threshold; it is minimum possible value of an attribute to trigger a sensor node. Hard threshold allows nodes transmit the event, if the event occurs in the range of interest. Therefore a significant reduction of the transmission delay occurs. Unless a change of minimum soft threshold occurs, the nodes don't send a new data packet. Employing soft threshold prevents from the redundant data transmission. Since the protocol is to be responsive to the sudden changes in the sensed attribute, it is suitable for time-critical applications.

A. Manjeshwar and D. P. Agarwal [8] proposed AdaPtive Threshold sensitive Energy Efficient sensor Network Protocol (APTEEN) protocol in 2002. The protocol is an extension of TEEN aiming to capture both time-critical events and periodic data collections. The network architecture is same as TEEN. After forming clusters the cluster heads broadcast attributes, the threshold values, and the transmission schedule to all nodes. Cluster heads are also responsible for data aggregation in order to decrease the size data transmitted so energy consumed. According to energy dissipation and network lifetime, TEEN gives better performance than LEACH and APTEEN because of the decreased number of transmissions. The main drawbacks of TEEN and APTEEN are overhead and complexity of forming clusters in multiple levels, implementing threshold-based functions and dealing with attribute based naming of queries.

In 2004, G. Smaragdakis, I. Matta and A. Bestavros [9] proposed Stable Election Protocol (SEP) protocol. This protocol is an extension to the LEACH protocol. It is a heterogeneous aware protocol, based on weighted election probabilities of each node to become cluster head according to their respective energy. This approach ensures that the cluster head election is randomly selected and distributed based on the fraction of energy of each node assuring a uniform use of the nodes energy. In this protocol, two types of nodes (two tier in-clustering) and two level hierarchies were considered.

In 2005, M. Ye, C. Li, G. Chen and J. Wu [10] proposed Energy Efficient Clustering Scheme (EECS) protocol. It is novel clustering scheme for periodical data gathering applications for wireless sensor networks. It elects cluster heads with more residual energy through local radio communication. In the cluster head election phase, a constant number of candidate nodes are elected and compete for cluster heads according to the node residual energy. The competition process is localized and without iteration. The method also produces a near uniform distribution of cluster heads. Further in the cluster formation phase, a novel approach is introduced to balance the load among cluster heads. But on the other hand, it increases the requirement of global knowledge about the distances between the cluster-heads and the base station.

In 2006, Q. Li, Z. Qingxin and W. Mingwen [11] proposed Distributed Energy Efficient Clustering Protocol (DEEC) protocol. This protocol is a cluster based scheme for multi level and two level energy heterogeneous wireless sensor networks. In this scheme, the cluster heads are selected using the probability based on the ratio between residual energy of each node and the average energy of the network. The epochs of being cluster-heads for nodes are different according to their initial and residual energy. The nodes with high initial and residual energy have more chances of the becoming cluster heads compared to nodes with low energy.

O. Younis and S. Fahmy proposed [4] Hybrid Energy Efficient Distributed clustering Protocol (HEED) protocol in 2004. It extends the basic scheme of LEACH by using residual energy as primary parameter and network topology features (e.g. node degree, distances to neighbors) are only used as secondary parameters to break tie between candidate cluster heads, as a metric for cluster selection to achieve power balancing. The clustering process is divided into a number of iterations, and in each iterations, nodes which are not covered by any cluster head double their probability of becoming a cluster head. Since these energy-efficient clustering protocols enable every node to independently and probabilistically decide on its role in the clustered network, they cannot guarantee optimal elected set of cluster heads.

\section{CLUSTER FORMATION OF HEED PROTOCOL}

In this section, we describe the network model. Assume that there are $\mathrm{N}$ sensor nodes, which are randomly dispersed within a $100 \mathrm{~m} * 100 \mathrm{~m}$ square region (Figure 1). Following assumptions are made regarding the network model is:

1. Nodes in the network are quasi-stationary.

2. Nodes locations are unaware i.e. it is not equipped by the GPS capable antenna.

3. Nodes have similar processing and communication capabilities and equal significance.

4. Nodes are left unattended after deployment.

Cluster head selection is primarily based on the residual energy of each node. Since the energy consumed per bit for sensing, processing, and communication is typically known, and hence residual energy can be estimated. Intra cluster communication cost is considered as the secondary parameter to break the ties. A tie means that a node might fall within the range of more than one cluster head. 


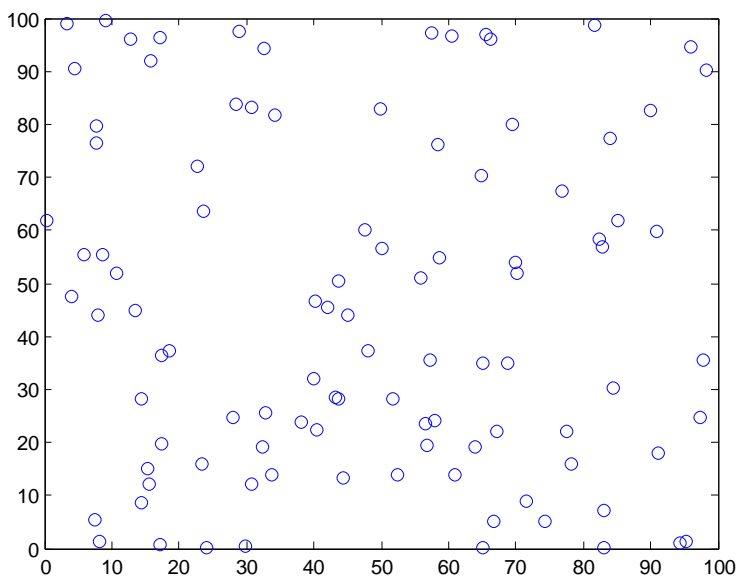

Figure 1. Random Deployment of 100 Sensor Nodes

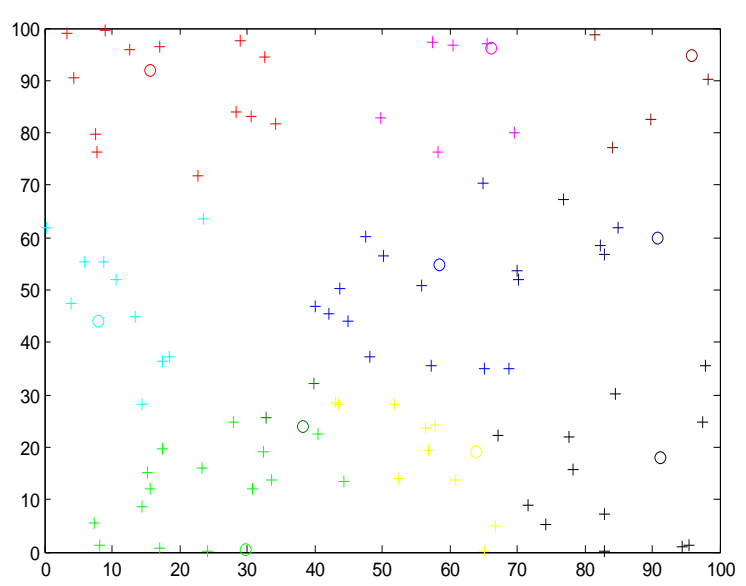

Figure 2. Clusters Formation by HEED protocol

When there are multiple candidate cluster heads, the cluster head yielding lower intra-cluster communication cost are favored. The secondary clustering parameter, intra-cluster communication cost, is a function of (i) cluster properties, such as cluster size, and (ii) whether or not variable power levels are permissible for intra-cluster communication. If the power level used for intracluster communication is fixed for all nodes, then the cost can be proportional to (i) Nodedegree, if the requirement is to distribute load among cluster heads, or (ii) 1 Wode Degree, if the requirement is to create dense clusters. This means that a node joins the cluster head with minimum degree to distribute cluster head load or joins the one with maximum degree to create dense clusters.

Each node performs neighbor discovery, and broadcasts its cost to the detected neighbors. Each node sets its probability of becoming a cluster head, CHprob, as follows:

$c H p r o b=\max \left(\right.$ cprob $\&\left(\frac{\text { Enesidwail }}{\text { Emax }}\right)$ opmin $)$

Where, Cprob is the initial percentage of cluster heads among $\mathrm{n}$ nodes (it was set to 0.05), while Eresidual and Emax are the residual and the maximum energy of a node (corresponding to the fully charged battery), respectively. The value of CHprob is not allowed to fall below the threshold pmin (i.e. $10^{-4}$ ). The clusters formation by HEED protocol is shown in figure 2 .

\section{HETROGENOUS NETWORK MODEL}

In 2-level H-HEED protocol, two types of sensor nodes, i.e., the advanced nodes and normal nodes are used. Let us assume there are ' $\mathrm{N}$ ' numbers of sensor nodes deployed in a field. $E_{0}$ is the initial energy of the normal nodes, and $m$ is the fraction of the advanced nodes, which own $a$ times more energy than the normal ones. Thus there are $m * N$ advanced nodes equipped with initial energy of $E_{0} *(1+a)$, and $(1-m) \& N$ normal nodes equipped with initial energy of $E_{0}$. The total initial energy of the network [9] is given by:

$$
\begin{gathered}
E_{\text {total }}=N \otimes(1-m) \otimes E_{0}+N \otimes m * E_{0} *(1+a) \\
=N \otimes E_{0} *(1+a m)
\end{gathered}
$$

So, this type of networks has am times more energy and virtually am more nodes.

In 3-level H-HEED protocol, there are three types of sensor nodes, i.e. the super nodes, advanced nodes and the normal nodes. Let $m$ be the fraction of the total number of nodes $N$, and $m_{0}$ is the percentage of the total number of nodes $N * m$ which are equipped with $\beta$ times more energy than the normal nodes, called as the super nodes, the number is $N * m * m_{0}$. The rest $N *$ $m *\left(1-m_{0}\right)$ nodes are having a times more energy than the normal nodes, being called as advanced nodes and the remaining $N *(1$ $m$ ) nodes are the normal nodes. $E_{0}$ is the initial energy of the normal nodes. The energy of the each super node is $E_{0} *(1+\beta)$ and the energy of each advanced node is $E_{0} \otimes(1+a)$.

The total energy of the networks $[13,14]$ is given by:

$$
\begin{aligned}
& E_{\text {total }}=N \otimes(1-m) \Leftrightarrow E_{0}+N \& m \&\left(1-m_{0}\right) * E_{0} * \\
& (1+a)+N \leqslant m \leqslant m_{0} * E_{0} *(1+\beta) \\
& E_{\text {total }}=\mathbb{N} \Leftrightarrow E_{0} \otimes\left(1+m *\left(a+m_{0} * \beta\right)\right)
\end{aligned}
$$

So, the total energy of the network is increased by the factor of $(1+m \&(a+m p \beta))$.

In multi-level H-HEED protocol, initial energy of sensor nodes is randomly distributed over the close set $\left[E_{0}, E_{0} *\left(1+a_{\max }\right)\right]$, where $E_{0}$ is the lower bound and $a_{\max }$ determine the value of the maximal energy. Initially, the node $s_{i}$ is equipped with initial energy of $E_{0} \&\left(1+a_{i}\right)$, which is $a_{i}$ times more energy than the lower bound $E_{0}$. The total initial energy of the network [11] is given by:

$$
\begin{aligned}
& E_{\text {total }}=\sum_{i=1}^{W} E_{0} \otimes\left(1+a_{i}\right) \\
& =E_{0} *\left(N+\sum_{i=1}^{W} a_{i}\right)
\end{aligned}
$$


During Cluster formation phase, every node will have its own Emax value in case of heterogeneity while computing the cluster head probability of the sensor node.

\section{SIMULATION RESULTS}

The simulation is done in Matlab. Let us assume the heterogeneous sensor network with 100 sensor nodes are randomly distributed in the $100 \mathrm{~m} * 100 \mathrm{~m}$ area. The base station is located at the centre $(50,50)$. We have set the minimum probability for becoming a cluster head (pmin) to 0.0001 and initially the cluster head probability for all the nodes is 0.05 . The parameters used in our simulation are listed in the Table 1.

TABLE 1: Simulation Parameters

\begin{tabular}{|l|l|}
\hline Parameters & Values \\
\hline Sink & At (50,50) \\
\hline Threshold distance, $\mathrm{d}_{0}$ & $70 \mathrm{~m}$ \\
\hline Cluster Radius & $25 \mathrm{~m}$ \\
\hline $\begin{array}{l}\text { Energy consumed in the electronics } \\
\text { circuit to transmit or receive the } \\
\text { signal, } E_{\text {elec }}\end{array}$ & $50 \mathrm{~nJ} / \mathrm{bit}$ \\
\hline $\begin{array}{l}\text { Energy consumed by the amplifier to } \\
\text { transmit at a short distance, } \mathrm{E}_{\mathrm{fs}}\end{array}$ & $10 \mathrm{pJ} / \mathrm{bit} / \mathrm{m}^{2}$ \\
\hline $\begin{array}{l}\text { Energy consumed by the amplifier to } \\
\text { transmit at a longer distance, } \mathrm{E}_{\mathrm{mp}}\end{array}$ & $0.0013 \mathrm{pJ} / \mathrm{bit} / \mathrm{m}^{4}$ \\
\hline Data Aggregation Energy, $\mathrm{E}_{\mathrm{DA}}$ & $5 \mathrm{~nJ} / \mathrm{bit} / \mathrm{signal}^{4}$ \\
\hline Message Size & $4000 \mathrm{bits}$ \\
\hline Initial Energy, $\mathrm{E}_{0}$ & $0.5 \mathrm{~J}$ \\
\hline
\end{tabular}

In the analysis, we use the same energy model as proposed in [12]. In the process of transmitting an 1-bit message over a distance $\mathrm{d}$, the energy expended by the radio is given by:

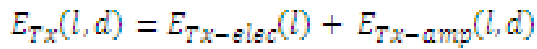

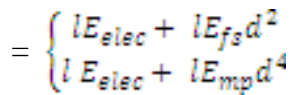

And to receive the message, the radio expends:

$$
E_{R x}(\mathbb{D})=E_{\text {Ru-eled }}(\mathbb{D})=\mathbb{R} E_{\text {elat }}
$$

The electronics energy, $E_{\text {eles }}$, depends on factors such as the digital coding, modulation, filtering, and spreading of the signal, whereas the amplifier energy, $E_{f g} d^{2}$ or $E_{m g} d^{4}$, depends on the distance to the receiver and the acceptable bit-error rate.

There are other factors like noise, physical obstacles or collision may affect the received power are ignored. We have introduced the advanced nodes to the HEED protocol, so as to assess the performance of HEED protocol in the presence of heterogeneity.

Let us consider the case for 2-level H-HEED, 30\% of the nodes are advanced nodes $(\mathrm{m}=0.3)$ and equipped with $150 \%$ more energy than normal nodes $(a=1.5)$. For 3-level H-HEED, $30 \%$ of the nodes are advanced nodes and $20 \%$ of the nodes are super nodes are equipped with $150 \%$ and $300 \%$ more energy than the normal nodes $\left(\mathrm{a}=1.5\right.$ and $\mathrm{b}=3, \mathrm{~m}=0.5$ and $\left.\mathrm{m}_{0}=0.4\right)$. For multilevel H-HEED, each node in the sensor network is randomly assigned different energy between a closed set $[0.5,2]$.

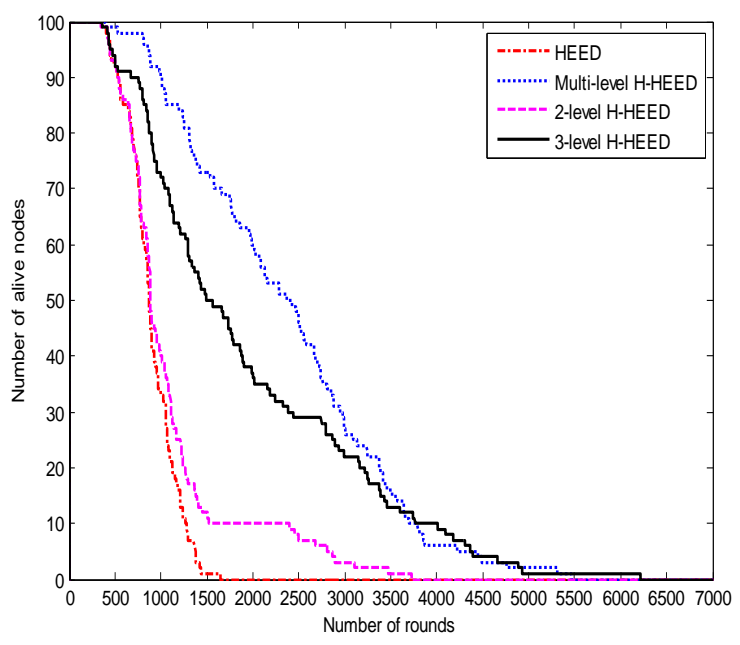

Figure 3 The Number of Alive Nodes per Round

In Fig. 3, a detailed view of the behavior of HEED and H-HEED protocol is illustrated; it shows the number of alive nodes per round. The number of nodes die in HEED is more than H-HEED over the same number of rounds. The number of normal nodes dies very fast and as a result the sensing field becomes sparse very fast. On the other hand, advanced nodes and super nodes die in a very slow fashion. But in multi-level H-HEED, all the sensor nodes are having different energy as a result nodes will die randomly. In this we can say that multi-level H-HEED prolongs lifetime and shows better performance than other level of $\mathrm{H}$ HEED and HEED protocol.

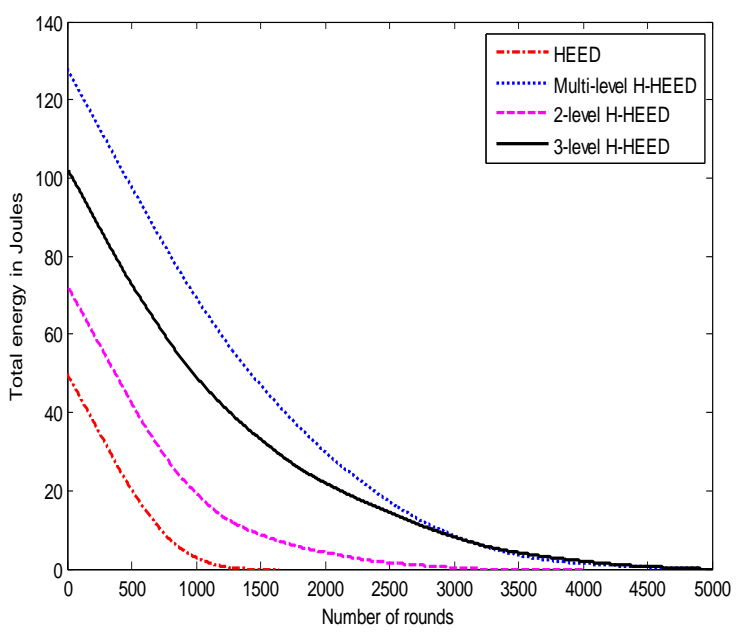

Figure 4 The total Remaining Energy in each Round

Figure 4 represents the total remaining energy of the network in each round. In this both HEED and H-HEED, the energy depletes very fast at constant rate. We can conclude that both 3-level $\mathrm{H}$ HEED and multi-level H-HEED is more energy efficient. 


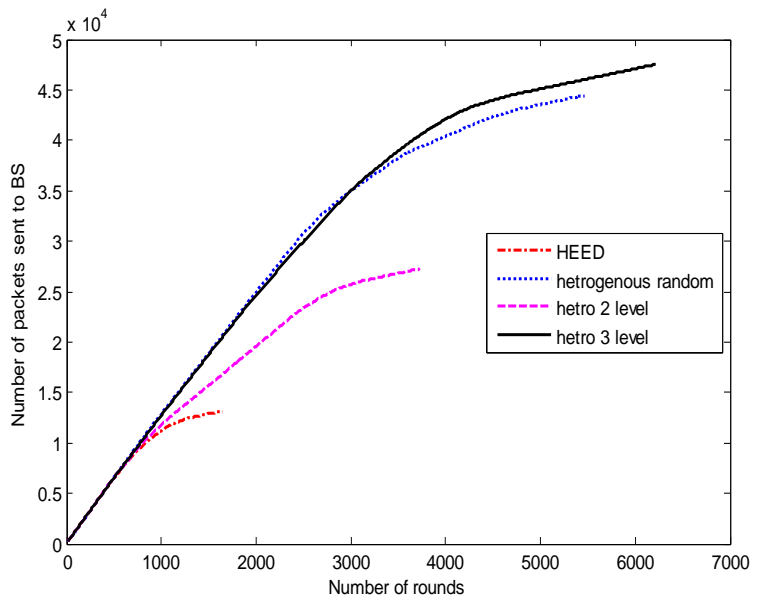

Figure 5 The Number of Packets Sent to the BS in each Round

Figure 5 represents the number of packets sent to the BS in each round. In this, more number of packets is sent in the H-HEED in comparison with HEED, as advanced nodes and super nodes will be having more probability of becoming the cluster heads, due to more residual energy so more number of packets will be sent to the base station. Thus, the H-HEED sends more effective data packets to the base station.

\section{CONCLUSION}

In this paper, H-HEED protocol is proposed for heterogeneous wireless sensor network. In this, we introduced different level of heterogeneity: 2-level, 3-level and multi-level in terms of the node energy. We have evaluated the performance of the proposed H-HEED with HEED protocol using Matlab. It is observed that there is significant improvement in the lifetime in case of $\mathrm{H}$ HEED protocol in comparison with HEED protocol because the number of rounds is maximum with multi-level H-HEED.

\section{REFERENCES}

[1] Marcos, Diogenes. 2003. Survey on Wireless Sensor Network Devices. IEEE.

[2] V. Raghunathan, C. Schurgers, Park. S, and M. B. Srivastava. 2002. Energy-aware wireless microsensor networks. IEEE Signal Processing Magazine, Volume: 19 Issue: 2, Page(s): $40-50$.

[3] I. Akyildiz et al. 2002. A Survey on Sensor Networks. IEEE Commun. Mag., vol. 40, no. 8, pp. 102-14.

[4] Ossama Younis and Sonia Fahmy. 2004. Distributed Clustering in Ad-hoc Sensor Networks: A Hybrid, EnergyEfficient Approach. In Proceedings of IEEE INFOCOM, Hong Kong, an extended version appeared in IEEE Transactions on Mobile Computing, 3(4).
[5] W. Heinzelman, A. Chandrakasan and H. Balakrishnan. 2000. Energy-Efficient Communication Protocol for Wireless Microsensor Networks. Proceedings of the 33rd Hawaii International Conference on System Sciences (HICSS '00).

[6] S. Lindsey, C. Raghavendra. 2002. PEGASIS: PowerEfficient Gathering in Sensor Information Systems. IEEE Aerospace Conference Proceedings, Vol. 3, 9-16 pp. 11251130.

[7] A. Manjeshwar and D. P. Agarwal. 2001. TEEN: a routing protocol for enhanced efficiency in wireless sensor networks. In 1st International Workshop on Parallel and Distributed Computing Issues in Wireless Networks and Mobile Computing.

[8] A. Manjeshwar and D. P. Agarwal. 2002. APTEEN: A hybrid protocol for efficient routing and comprehensive information retrieval in wireless sensor networks. Parallel and Distributed Processing Symposium, Proceedings International, IPDPS, pp. 195-202.

[9] G. Smaragdakis, I. Matta, A. Bestavros. 2004. SEP: A Stable Election Protocol for clustered heterogeneous wireless sensor networks. In Second International Workshop on Sensor and Actor Network Protocols and Applications (SANPA).

[10] M. Ye, C. Li, G. Chen, J. Wu. 2005. EECS: an energy efficient cluster scheme in wireless sensor networks. In IEEE International Workshop on Strategies for Energy Efficiency in Ad Hoc and Sensor Networks (IEEE IWSEEASN-2005), Phoenix, Arizona, April 7-9.

[11] Q. Li, Z. Qingxin, and W. Mingwen. 2006. Design of a distributed energy efficient clustering algorithm for heterogeneous wireless sensor networks. Computer Communications, vol. 29, pp. 2230-7.

[12] W.R. Heinzelman, A.P. Chandrakasan, H. Balakrishnan. 2002. An application-specific protocol architecture for wireless microsensor networks. IEEE Transactions on Wireless Communications 1 (4) 660-670.

[13] R. B. Patel, T. S. Aseri, Dilip Kumar. 2009. EEHC: Energy efficient heterogeneous clustered scheme for wireless sensor networks. International Journal of Computer Communications, Elsevier, 32(4): 662-667.

[14] Yingcghi Mao, Zhen Liu, Lili Zhang and Xiaofang Li. 2009. An Effective Data Gathering Scheme in Heterogeneous Energy Wireless Sensor Network. In International Conference on Computational Science and Engineering. 